\title{
Intravitreal ocriplasmin: A breakthrough in the treatment of vitreomacular traction?
}

\author{
Anna E. Mec-Słomska ${ }^{1, A-D}$, Joanna Adamiec-Mroczek2, B, E, F, Ewa Kuźmicz ${ }^{3, \text { B }}$, Marta Misiuk-Hojło2, E \\ 1 Provincial Hospital Center, Jelenia Góra, Poland \\ ${ }^{2}$ Department and Clinic of Ophthalmology, Wroclaw Medical University, Poland \\ ${ }^{3}$ Medical Center Aldemed, Zielona Góra, Poland \\ A - research concept and design; B - collection and/or assembly of data; C - data analysis and interpretation; \\ $D$ - writing the article; $E$ - critical revision of the article; $F$ - final approval of article
}

Address for correspondence

\section{Anna Mec-Słomska}

E-mail:annamec@op.pl

Funding sources

None declared

\section{Conflict of interest}

None declared

Received on March 11, 2015

Revised on November 30, 2015

Accepted on March 09, 2016

DOI

10.17219/acem/62122

\section{Copyright}

Copyright by Author(s)

This is an article distributed under the terms of the

Creative Commons Attribution Non-Commercial License

(http://creativecommons.org/licenses/by-nc-nd/4.0/)

\begin{abstract}
Vitreoretinal interface pathologies, such as vitreomacular traction syndrome, epiretinal membranes and macular holes are sight-threatening conditions and one of the important causes of vision defects and vision loss. To this date, vigilance with observation of how the vitreomacular traction resolves, or vitreoretinal surgery in more severe cases, were the only treatment options. Recent rapid progress in ophthalmology, especially in diagnostic and visualization techniques, provided better insight into the mechanisms taking place on the vitreoretinal surface, which enabled a more accurate selection of treatment options. Development of ophthalmic pharmacological procedures, such as treatment of vitreomacular traction syndrome with ocriplasmin, constitutes an innovative breakthrough in ophthalmology. The enzyme is a genetically engineered form of human plasmin, a component of blood coagulation cascade that has been envisioned for human therapy since 1950s. It has never been used for vitreolysis in ophthalmology before. The aim of this review is to analyze and compare therapeutic options for symptomatic vitreomacular adhesion and vitreoretinal traction, with particular emphasis on microplasmin. We reviewed the results of recent studies comparing ocriplasmin to other widespread treatment options, such as pars plana vitrectomy.
\end{abstract}

Key words: vitreoretinal surgery, optical coherence tomography, microplasmin, vitreous body, vitreous detachment. 
Rapid progress in retinal visualization techniques taking place during the last decade facilitated accurate diagnosis and selection of appropriate treatment methods. Introduction of optical coherence tomography (OCT) to the panel of standard retinal diagnostic tests with no doubt represents a milestone in ophthalmology. OCT, also referred to as optical biopsy, provides detailed insight into the retinal structure and vitreoretinal interface. As a result, various abnormalities that previously could not be visualized by means of ophthalmoscopy, such as vitreoretinal traction syndrome, can be expeditiously and accurately diagnosed. This is of vital importance as persistent adhesion and partial detachment of the posterior vitreous cortex that take place on the vitreoretinal surface are the leading cause of many vitreoretinal pathologies.

\section{Vitreous body and vitreoretinal interface pathologies}

Interaction between the retina and vitreous body vary by location. The vitreoretinal interface "glue like" proteins, i.e. laminin and fibronectin, fix collagen fibrils of the posterior vitreous body to the internal limiting membrane (ILM) of the retina. ${ }^{1}$ At the vitreous base, vitreous collagen fibers pass through the ILM and communicate directly with retinal collagen. ${ }^{2}$

Posterior vitreous detachment (PVD) from the ILM of the retina is a common condition in an ageing eye. Vitreous body liquefaction and its collapse that occur during the course of the ageing process lead to complete vitreoretinal separation in the majority of patients. PVD is a natural process and does not contribute to vision loss. ${ }^{3,4}$

Vitreous is most firmly attached to the retina at the vitreous base, optic disc, fovea and along the major retinal blood vessels. ${ }^{5}$ Perifoveal detachment with residual vitreofoveal adhesion is considered the first stage of PVD. ${ }^{6}$ Due to an incomplete posterior vitreous separation, some residual sites of persistent vitreoretinal adhesion (VA) can still be observed.

Vitreomacular adhesion (VMA) is defined as a perifoveal vitreous separation with remaining vitreomacular attachment and unperturbed foveal morphologic features. As long as adhesions of the vitreous to the retina do not transform into a pathological vitreoretinal traction (VRT), the condition is asymptomatic and does not result in any malformation or damage of the retinal architecture. ${ }^{4,7,8}$

Vitreomacular traction (VMT) is associated with anomalous posterior vitreous detachment accompanied by anatomic distortion of the fovea and intraretinal structural changes. The Vitreomacular Study Group proposed a classification of vitreomacular adhesions based on the diameter of the vitreous attached to the macular surface measured by OCT. Attachment of $1500 \mu \mathrm{m}$ or less is defined as a focal and attachment of more than $1500 \mu \mathrm{m}$ as broad. ${ }^{9}$ VRT occurs whenever the detaching posterior vitreous surface produces pulling forces on the areas of the retina with interface pathologies. Macula and fovea are particularly sensitive. VMT may cause microscopic damage of the retinal surface, which induces the healing process along with the formation of fibroglial scar tissue, proliferation of myofibrocytes and astrocytes. This eventually leads to the thickening of the ILM and epiretinal membranes. ${ }^{10-12}$

VMT is a potentially sight-threatening condition, manifesting itself as metamorphopsia, photopsia, blurred vision, decreased visual acuity and optical distortion. Escalating VMT may lead to various macular defects, such as foveal pseudocysts, macular edema (ME), macular holes $(\mathrm{MH})$, accumulation of subretinal fluid and more or less intense bleeding from damaged retinal vessels, retinoschisis or even tractional retinal detachment. It may also be associated with age-related macular degeneration (AMD). ${ }^{13}$ Furthermore, it has been suggested that VMA may be associated with the progression of diabetic retinopathy. ${ }^{14}$

$\mathrm{MH}$ is a full-thickness break in the retinal fovea, involving retinal layers from internal limiting membrane to outer segments of photoreceptors.

\section{Diagnosis and treatment of vitreoretinal interface pathologies}

Diagnosis of vitreoretinal interface pathologies includes ophthalmoscopy, biomicroscopy, visual function tests (such as Amsler test, Watzke-Allen test, laser beam test, microperimetry), USG A and B. Crucial state-of-theart imaging technologies, such as spectral domain OCT (SD-OCT) and spectral OCT imaging with confocal scanning laser ophthalmoscope (SLO) (SD-OCT/SLO), are the most sensitive diagnostic tools that improve visualization of the vitreoretinal surface.

Currently, a standard approach to the treatment of vitreomacular pathologies, such as symptomatic VMA and VMT, includes observation and watchful waiting or surgery. According to the general recommendations that have been published in 2014 and onwards, inter alia by Shao Lei and Wei Wenbin, patients with asymptomatic VMT should be observed for at least 2-3 months to see if a spontaneous PVD occurs, and surgery is recommended in the case of significant VMT-related disorders, such as ERM or MH. ${ }^{15}$

Typically observation and waiting are continued until VMT resolves spontaneously or until visual acuity of the patient decreases, visual symptoms become intolerable and macular defects progress. Under such circumstances, a decision to perform the intraocular surgery is made by a vitreoretinal surgeon.

However, other studies demonstrated that delayed vitrectomy and too long observation of patients with $\mathrm{MH}$ 
and VMT might result in a worse outcome than after a quick surgery. In a paper published in 2005, Jaycock et al. reported that a shorter time between the diagnosis and surgery of $\mathrm{MH}$ is associated with better postoperative visual acuity and anatomical closure rates: $95.2 \%$ for $\mathrm{MH}$ of less than 6 months duration, 91.7\% for those from 6 to 12 months duration, and $47.4 \%$ for those persisting longer than 1 year. $^{16}$ According to Theodossiadis et al., persistent traction on the macula may induce irreversible damage to the structure of retinal layers and induce ERM formation. This may be the reason behind incomplete functional and anatomical recovery of the retina after a delayed surgery. ${ }^{17}$

Pars plana vitrectomy (PPV) with membrane peeling relieves traction and leads to normalization of the macular architecture and function. ${ }^{8,13}$ Reconstitution of the macular neurosensory layers observed on post-operative OCT was identified as the most relevant parameter for visual improvement. ${ }^{18}$

The aim of the PPV procedure is to surgically detach the posterior surface of the vitreous from the retina, perform vitrectomy, ILM peeling, and thereby relieve vitreoretinal tractions.

Despite the significant progress in vitroretinal surgery since its introduction in early 1970s, it is still not free from severe complications. Use of modern equipment, smaller gauge systems, sutureless techniques, high-speed vitrectomes and chromovitrectomy make it possible to treat the majority of pathologies located at the vitreoretinal interface. ${ }^{19}$ However, although this technique is highly effective, represents a breakthrough in ophthalmology and as such is now commonly performed in ophthalmological centers, PPV always requires extreme caution and may be associated with complications.

The most serious adverse events associated with vitrectomy include endophthalmitis (observed in 0.018-0.23\% of the cases), bleeding (0.14-0.17\%), retinal detachment (in up to $10.9 \%$ of patients subjected to 20 - or 23 -gauge PPV), infrequent progression of a cataract that may develop in $42.5 \%$ of the vitrectomized patients, macular edema (5.5\%) and retinal breaks. ${ }^{20-25}$ Noticeably, surgical removal of macular tractions associated with mechanical induction of the posterior hylaoid face (PHF) separation represents an important risk factor for development of iatrogenic retinal breaks. The incidence of this complication is reported at up to $18.2 \%$ in the case of 23 -gauge PPV. ${ }^{26}$ This may be associated with the presence of VMTrelated abnormalities at the vitreoretinal interface.

\section{Pharmacological treatment of symptomatic VMA}

Research on simpler and more effective preventive measures that could be implemented at earlier stages of vitreoretinal junction pathologies stimulated prog- ress in pharmacotherapy. Pharmacological induction of PVD represents a breakthrough in minimizing the need for surgical procedures and the risk of related complications.

On October 17, 2012, the United States Food and Drug Administration (FDA) authorized ocriplasmin for the treatment of symptomatic vitreomacular adhesion, and on March 13, 2013 this agent was granted marketing authorization for the European Union states. Ocriplasmin is the first injectable drug approved to treat symptomatic VMA. But does it constitute a true breakthrough in ophthalmology?

Ocriplasmin, also referred to as microplasmin, is a truncated form of plasmin, an enzyme that has been envisioned for human therapy since 1950s and is a component of the blood coagulation cascade. Plasmin and ocriplasmin have been studied to be effective in vitreolysis. ${ }^{27,28}$ Administration of ocriplasmin into the vitreous cavity was shown to result in a dose- and time-dependent posterior vitreous detachment. ${ }^{29}$

Ocriplasmin, a recombinant human protein derived from the cells of yeast Pichia pastoris with a recombinant DNA technology, is a variant of human plasmin with retained protease activity. It is active against the substrates that play an important role in the vitreous structure and vitreoretinal interface, such as collagen, fibronectin and laminin. Laminin and fibronectin are involved in attaching the posterior vitreous to the retina. Microplasmin belongs to the serine protease family; it degrades the protein scaffold linking the vitreous to the retina, induces vitreous liquefaction and vitreoretinal separation. ${ }^{28,30}$ When administered at a therapeutic dose, ocriplasmin does not induce any morphological or functional changes of the retina.

The enzyme consists of 2 polypeptide chains linked by sulfide bonds. The longer chain has 4 additional disulfide bonds. Due to its proteolytic and autolytic properties under physiological $\mathrm{pH}$, microplasmin is only moderately stable upon injection and undergoes a rapid degradation. Therefore, the time of its activity is fairly limited. ${ }^{27,28}$ Its biological activity within the vitreous cavity can be observed no longer than several days post-injection. ${ }^{31}$

The drug is available in solution for injection. It is designed for intravitreal use under aseptic controlled conditions. The recommended dose is $0.125 \mathrm{mg}$ administered by pars plana intravitreal injection to the affected eye as a single injection. Ophthalmological monitoring for potential side effects should follow the procedure. Administration to both eyes, as well as repeated administration to the same eye, is not recommended, as there is insufficient evidence of the safety of such approach.

Although the drug seems to be highly promising as a treatment for VMA that may restore anatomical and functional conditions with a minimally invasive and less traumatic procedure than vitrectomy, its use still raises some controversies. 
Ocriplasmin is indicated for the treatment of vitreomacular traction (VMT) in adults, also associated with a macular hole of $\leq 400$ microns in diameter. Patients with smaller baseline diameters of VMA ( $\leq 1500$ microns) are more likely to benefit from the treatment than those with diameters of $>1500$ microns. ${ }^{32}$ Analysis of the vitreoretinal interface abnormalities, conducted within the framework of 2 phase III studies on intravitreal ocriplasmin, demonstrated that ERM may decrease the efficacy of ocriplasmin efficacy, perhaps due to the increase in the strength of fibrocellular organization and contraction. ${ }^{28,32-34}$ The best outcomes are observed in younger ( $<65$ years) phakic patients, as well as in individuals in whom VMT is associated with $\mathrm{MH}$ of $<250$ microns in diameter. ${ }^{32}$ Consequently, indications for ocriplasmin treatment are currently limited, and appropriate qualification of patients represents a key to a successful outcome.

In a controlled randomized multicenter pivotal study on enzymatic vitreolysis with ocriplasmin for VMT and $\mathrm{MH}$ demonstrated that vitreomacular adhesion resolved in $26.5 \%$ and $10 \%$ of ocriplasmin- and placebo-injected eyes, respectively. Total PVD occurred in $13.4 \%$ and $3.7 \%$ of ocriplasmin- and placebo-injected eyes, respectively, and vision improved by 3 or more lines in $12.3 \%$ of ocriplasmin-injected eyes. Nonsurgical closure of $\mathrm{MH}$ by day 28 was achieved in $40.6 \%$ of ocriplasmin-injected eyes and in only $10.6 \%$ of eyes injected with placebo. ${ }^{28}$ Although these outcomes are highly impressive, the results of recent research on the application of ocriplasmin for the treatment of stage $2 \mathrm{MH}$ imply that the closure rates may be lower and sporadically the holes that failed to close after ocriplasmin injection may even enlarge. ${ }^{35}$

The results of phase III trials imply that ocriplasmin is usually well tolerated. The main adverse events, such as vitreous floaters, were reported in $16.8 \%$, progression of cataract in $8.2 \%$, and mild or transient intraocular inflammation in about $7 \%$ of ocriplasmin-treated patients. ${ }^{28}$ Also other transient mild ocular adverse events, such as eye pain and decreased visual acuity, were observed after the drug was administered. The incidence of post-injection retinal detachment and retinal tear was similar as in the placebo group. ${ }^{28}$

Owing to the proteolytic properties of microplasmin, this enzyme may also target other intraocular structures that contain fibronectin, laminin or collagen IV, e.g. the lenticular zonules. This may result in lens subluxation and phacodonesis. Although studies using scanning an electron microscope did not reveal any post-injection damage to the ciliary body and zonules, a single case of lens subluxation following intravitreal ocriplasmin injection has been reported in a participant of a phase II trial. ${ }^{27,36,37}$

Ocriplasmin may also exert a diffuse enzymatic effect, which is not limited solely to the macular region but involves the entire retina, including photoreceptors and retinal pigment epithelium (RPE). Importantly, mi- croplasmin's target, laminin is also present in multiple retinal layers, including photoreceptors and retinal pigment epithelium. This may explain the etiology of a few reported cases in which an acute panretinal dysfunction and vision loss with severely reduced electroretinography responses were observed after intravitreal ocriplasmin injection. ${ }^{38,39}$

Furthermore Silva et al. reported 2 cases in which the formation of retinal breaks leading to retinal detachment (involving macula in one patient) was documented on continued follow-up. In both these cases, retinal breaks did not result from incorrect ocriplasmin injection, but rather were the consequence of successful releasing VMT and remaining VRT on the periphery of the retina. ${ }^{40}$

To this date, we lack sufficient evidence regarding the application of microplasmin in some specific clinical situations, e.g. concomitantly to anti-VEGF therapy, in patients with large-diameter macular holes, individuals with high myopia, aphakia, history of rhegmatogenous retinal detachment, lens zonule instability, recent ocular surgery, proliferative diabetic retinopathy, ischemic retinopathies, retinal vein occlusions, exudative AMD, vitreous hemorrhage, infants and children scheduled for vitrectomy. Therefore, ocriplasmin is not recommended in such cases and considered solely as an experimental treatment.

Owing to the innovative character of the treatment and the fact that ocriplasmin is a subject of many ongoing and planned clinical trials, this agent is still monitored by various bodies, including the European Medicines Agency.

The high cost of ocriplasmin therapy may also constitute a significant obstacle on the route to considering it a widespread standard treatment. Considering the fact that VMA resolved in only $26.5 \%$ of ocriplasmin-treated patients participating in clinical trials and the drug itself is costly, PPV may turn out to be more cost-effective. ${ }^{28}$

\section{Conclusion}

Despite all the concerns regarding ocriplasmin treatment, it doubtlessly constitutes an alternative to vitrectomy and expectant management in patients with vitreomacular interface pathologies. Nonsurgical introduction of PVD with microplasmin is less traumatic and may eliminate major risks inherent to surgical approach. It also provides a more physiological state of the retina without exposing it potential side effects, such as retinal breaks and iatrogenic macular damage that may occur during the ILM peeling and introduction of PVD. Furthermore, enzymatic vitreolysis also allows for earlier intervention and prevents consequences of VMT. Although further research is still needed, the discovery and introduction of ocriplasmin treatment constitute a significant step forward toward a minimally invasive surgery and a breakthrough in the treatment of VMT. 


\section{References}

1. Matsumoto B, Blanks JC, Ryan SJ. Topographic variations in the rabbit and primate internal limiting membrane. Invest Ophthalmol Vis. Sci. 1984;25(1):71-82.

2. Wang J, McLeod D, Henson DB, Bishop PN. Age-dependent changes in the basal retinovitreous adhesion. Invest Ophthalmol Vis Sci. 2003;44(5):1793-1800.

3. Sebag J. Balazs EA. Morphology and ultrastructure of human vitreous fibres. Invest Ophthalmol Vis Sci. 1989;30:1867-1871.

4. Steel DHW. Lotery AJ. Idiopathic vitreomacular traction and mac ular hole: A comprehensive review of pathophysiology, diagnosis, and treatment. Eye. 2013;Suppl 1:S1-21. doi: 10.1038/eye.2013.212.

5. Sebag J. Ageing of the vitreous. Eye. 1987;1:254-262.

6. Uchino $E$, Uemura $A$, Ohba N. Initial stages of posterior vitreous detachment in healthy eyes of older persons evaluated by optical coherence tomography. Arch Ophthalmol. 2001;119(10):1475-1479.

7. Johnson MW. Perifoveal vitreous detachment and its macular complications. Trans Am Ophthalmol Soc. 2005;103:537-567.

8. Paolo Carpineto, Luca Di Antonio, Agbeanda Aharrh-Gnama, Vincenzo Ciciarelli, Leonardo Mastropasqua. Diagnosing and treating vitreomacular adhesion. Eur Ophthal Rev. 2011,5(1):69-73.

9. Duker JS, Kaiser PK, Binder S, et al. The International Vitreomac ular Traction Study Group classification of vitreomacular adhesion, traction, and macular hole. Ophthalmology. 2013;120(12):2611-2619. doi: 10.1016/j.ophtha.2013.07.042.

10. Sebag J. Molecular biology of pharmacologic vitreolysis. Trans Am Ophthalmol Soc. 2005;103:473-494.

11. Smiddy WE, Green WR, Michels RG, de la Cruz Z. Ultrastructural studies of vitreomacular traction syndrome. Am. J. Ophthalmol. 1989;107(2):177-185.

12. Shinoda K, Hirakata A, Hida T et al. Ultrastructural and immunohistochemical findings in five patients with vitreomacular traction syndrome. Retina. 2000;20(3):289-293.

13. Mojana F, Cheng L, Bartsch DU, et al. The role of abnormal vitreomacular adhesion in age-related macular degeneration: Spectral optical coherence tomography and surgical results. Am J Ophthalmol. 2008;146(2):218-227. doi: 10.1016/j.ajo.2008.04.027.

14. Jackson TL, Nicod E, Angelis A, et al. Vitreous attachment in agerelated macular degeneration, diabetic macular edema, and retinal vein occlusion: A systematic review and metaanalysis. Retina. 2013;33(6):1099-1108. doi: 10.1097/IAE.0b013e31828991d6.

15. Shao Lei, Wei Wenbin. Vitreomacular traction syndrome. Chin Med J. 2014;127(8): 1566-1571.

16. Jaycock PD, Bunce C, XingW, et al. Outcomes of macular hole surgery: Implications for surgical management and clinical governance. Eye. 2005;19:879-884.

17. Theodossiadis GP, Grigoropoulos VG, Theodoropolou S, Datseris I, Theodossiadis PG. Spontaneous resolution of vitreomacular traction demonstrated by spectral-domain optical coherence tomography. Am J Ophthalmol. 2014;157:842-851.

18. Sayegh RG, Georgopoulos M, Geitzenauer W, Simader C, Kiss C, Schmidt-Erfurth U. High-resolution optical coherence tomography after surgery for vitreomacular traction Ophthalmology. 2010;117(10):2010-2017 doi: 10.1016/j.ophtha.2010.01.041.

19. Rizzo S, Patelli F, Chow DR. Essentials in Ophthalmology. Vitreo-retinal Surgery. Springer;2009:69-87.

20. Kunimoto DY, Kaiser RS, Willis Eye Research Service: Incidence of Endophthalmitis after 20- and 25- Gauge Vitrectomy. Ophthalmology. 2007;114(12):2133-2137.

21. Sharma T, Virdi DS, Parikh S, et al. A case-control study of suprachoroidal hemorrhage during pars plana vitrectomy. Ophthalmic Surg Lasers.1997;28(8):640-644.
22. Ghoraba $\mathrm{HH}$, Zayed Al. Suprachoroidal hemorrhage as a complication of vitrectomy. Ophthalmic Surg Lasers. 2001;32(4):281-288.

23. De Nie KF, Crama N, Tilanus MA, Klevering BJ, Boon CJ. Pars plana vitrectomy for disturbing primary vitreous floaters: Clinical outcome and patient satisfaction. Graefes Arch Clin Exp Ophthalmol. 2013;251(5):1373-1382.

24. Chung CP, Hsu SY, Wu WC. Cataract formation after pars plana vitrectomy. Kaohsiung J Med Sci. 2001;17(2):84-89.

25. Mason JO $3^{\text {rd }}$, Neimkin MG, Mason JO $4^{\text {th }}$, et al. Safety, efficacy, and quality of life following sutureless vitrectomy for symptomatic vitreous floaters. Retina. 2014;34(6):1055-1061.

26. Rahman R, Murray CD, Stephenson J. Risk factors for iatrogenic retinal breaks induced by separation of posterior hyaloid face during 23-gauge pars plana vitrectomy. Eye. 2013;27(5):652-656. doi: 10.1038/eye.2013.6.

27. de Smet MD, Valmaggia C, Zarranz-Ventura J, Willekens B. Microplasmin: Ex vivo characterization of its activity in porcine vitreous. Invest Ophthalmol Vis Sci. 2009;50:814-819.

28. Stalmans P, Benz MS, Gandorfer A, et al. Enzymatic vitreolysis with ocriplasmin for vitreomacular traction and macular holes. $N$ Engl J Med. 2012;367:606-615. doi: 10.1056/NEJMoa1110823.

29. Stalmans $P$, de Laey $C$, de Smet $M$, van Dijkman E, Pakola S. Intravitreal injection of microplasmin for treatment of vitreomacular adhesion: Results of a prospective, randomized, sham-controlled phase II trial (the MIVI-IIT trial). Retina. 2010;30:1122-1127.

30. European Medicines Agency. Assessment report: Jetrea; 17 January 2013 EMA/CHMP/74766/2013. Committee for Medicinal Products for Human Use (CHMP).

31. de Smet MD, Jonckx B, Vanhove M, van Calster J, Stalmans P, Stassen JM. Pharmacokinetics of ocriplasmin in vitreous. IOVS. 2012;53(13).

32. Haller JA, Stalmans P, Benz MS, et al. Efficacy of intravitreal ocriplasmin for treatment of vitreomacular adhesion: Subgroup analyses from two randomized trials. Ophthalmology. 2015;122(1):117122. doi: 10.1016/j.ophtha.2014.07.045.

33. DeCroos FC, Toth CA, Folgar FA, et al. Characterization of vitreoretinal interface disorders using OCT in the interventional phase 3 trials of ocriplasmin. Invest Ophthalmol Vis Sci. 2012;53(10):6504-6511.

34. Song SJ, Smiddy WE. Ocriplasmin for symptomatic vitreomacular adhesion: An evidence-based review of its potential. Core Evid. 2014;9:51-59. Published online Mar 21, 2014. doi: 10.2147/CE.S39363

35. Miller JB, Kim LA, Wu DM, Vavvas DG, Eliott D, Husain D. Ocriplasmin for treatment of stage 2 macular holes: Early clinical results. Ophthal Surg Lasers Imaging Retina. 2014;45(4):293-297. doi: 10. 3928/23258160-20140709-05.

36. Song SJ, Smiddy WE. Ocriplasmin for symptomatic vitreomacular adhesion: An evidence-based review of its potential. Core Evid. 2014;9:51-59. doi: 10.2147/CE.S39363.

37. US Food and Drug Administration. Jetrea (ocriplasmin) Intravitreal Injection Medical Review. October 17, 2012. http://www.accessdata.fda.gov/drugsatfda docs/nda/2012/1254220rig1s000MedR.pdf. Accessed May 22, 2013.

38. Tibbetts MD, Reichel E, Witkin AJ. Vision loss after intravitreal ocriplasmin: Correlation of spectral-domain optical coherence tomography and electroretinography. JAMA Ophthalmol. 2014;132(4):487490. doi: 10.1001/jamaophthalmol.2013.8258.

39. Fahim AT, Khan NW, Johnson MW. Acute panretinal structural and functional abnormalities after intravitreous ocriplasmin injection. JAMA Ophthalmol. 2014;132(4):484-486. doi: 10.1001/jamaophthalmol.2013.8142.

40. Silva RA, Moshfeghi DM, Leng T. Retinal breaks due to intravitreal ocriplasmin. Clin Ophthalmol. 2014;8:1591-1594. doi: 10.2147/OPTH. S68037. 